\title{
DOING RESEARCH WITH ENUMERATORS
}

\section{WHY MASTER THIS SKILL?}

Data collection often requires the assistance of a team of data collectors, or enumerators, as they are usually called. This may include the following types of research:

- Household and other large-scale surveys; censuses;

- Qualitative research with large numbers of respondents.

Enumerators are typically asked to administer survey questionnaires or collect information using digital or paper collection modes that are designed to capture information around a research or evaluation question. It is essential that they are well managed and trained: if enumerators are not well chosen, prepared and motivated, this can have adverse effects on the efficiency of research and the quality of the data collected. This guideline outlines how to recruit, train and work with enumerators.

\section{RECRUITING ENUMERATORS}

\section{When are enumerators required?}

Enumerators are given research tools such as an interview guide, questionnaire or household survey and asked to collect information in a specific location or area from a certain number of individuals or households or groups. This may be necessary for one or both of the following reasons:

- the quantity of data required - Oxfam's large- $n$ Effectiveness Reviews, for example, usually survey hundreds of households or people;

- the type of data required - many research and evaluation projects rely on the local understanding of someone familiar with a given context to collect meaningful information, requiring, for example, specific linguistic, social and cultural attributes. 


\section{What to look for in enumerators}

\section{Basic skills}

The purpose of the project will determine the appropriate research tool(s) and method(s) enumerators will use to collect data. For example, many of Oxfam's Effectiveness Reviews require enumerators to administer quantitative household surveys that measure consumption or income, among other things. But enumerators can also administer qualitative research tools. This has been the case for example where Oxfam has used SenseMaker, which involves collecting short personal stories from respondents. Enumerators can use different modes to collect data, including paper, or mobile devices like tablets. Organizations like Oxfam increasingly use digital data collection to ensure high quality data. Which tools are used depends on the questions being addressed - but enumerator skills should be considered in this process.

Enumerators need to be able to critically appraise what information to collect (including what is considered a benchmark of high quality), and be able to do so practically, which relies on literacy and numeracy, and linguistic, social, and other skills.

\section{Context and identity}

It is always important to consider the socioeconomic status of enumerators (e.g. as trusted community members or members of a certain caste or class), their gender, marital status, age group, ethnicity or religion. Demographic and ethnic attributes can make respondents much more likely to share relevant, unbiased information openly.

For some types of research in particular, such as in areas affected by conflict or gender-based violence, identity politics matter. Even when training enumerators, the trainers' or evaluators' identity can affect enumerators' motivation and attitudes towards their work. For example, an unmarried, young woman training older married women of a different ethnic group to administer a survey about maternal health may not be taken seriously, or may be perceived as initimidating.

Where there is a risk of tension around identity, a trusted authority figure who has the respect of the group of enumerators should be asked to introduce and vouch for the trainer, and clarify the added value of the research.

\section{PREPARING THE ENUMERATORS}

\section{How to recruit enumerators}

How enumerators are recruited has important consequences for data collection. Sometimes organizations like Oxfam contract individuals directly; for example, because they are trusted community contacts. More often, Oxfam subcontracts an organization or company as intermediary through a formal tendering process. These intermediary organizations perform a range of tasks for the project in question, including choosing, hiring and monitoring enumerators for data collection. They are (partly) responsible for data quality and can be asked to conduct training. 


\section{Setting expectations}

It is important to set clear expectations for enumerators from the start, and to reiterate these over the course of the data collection.

\section{Workload and pay}

It is essential to communicate early on what enumerators are expected to complete, in what timeframe, and how they will be remunerated. Workload may be set as a minimum amount of time worked per day or number of interviews completed, ideally linked to a quality control mechanism whereby surveys or interviews need to meet certain quality standards to be part of the final sample. Depending on the length of data collection and whether any additional expenses like accommodation are included, enumerators can be paid upfront, throughout the research or on completion of the project.

\section{Roles, responsibilities and incentives}

To ensure enumerators and, if applicable, intermediaries work efficiently and to the best of their abilities, it helps to communicate the importance of their role. Enumerators need to understand why their work is vital and why they are best placed for acquiring information on a topic that matters, and that working to the best of their ability will increase the quality of the data being collected. In short, everybody involved should know who they are reporting to, and what they are responsible for.

To work as seamlessly as possible, it helps to try to understand intermediaries' and enumerators' specific incentives. Enumerators may only be motivated by financial remuneration. They may also have a personal or professional interest in the research questions being asked or the tools used to collect data, in which case they are likely to respond to a different set of encouragements. Or they may try to establish themselves as trusted professionals in a given context - this is often the case where there is a large pool and high demand for skilled enumerators, and/or where people are dependent on agencies like Oxfam as employers.

\section{Refining the research tool}

It is possible to teach enumerators how to administer a pre-set survey, such as a household survey measuring consumption. This usually requires standard training that can be transferred from one project context to the next - hence a structure that is easy to replicate and requires minimal input. On the other hand, it is also possible to involve enumerators deeply in co-constructing or refining the research tool at hand. For example, enumerators can help with the wording of questions, the accuracy of translations, and more general aspects of the research (e.g., through pilot activities that include a cycle of feedback from enumerators). Moreover, research that needs to stay agile in responding to the local context may benefit from advice from local enumerators, for example relating to sampling and location constraints linked to safety in risky situations. How involved enumerators are expected to be should be communicated clearly and early. 


\section{TRAINING DO'S AND DON'TS}

Training will be different according to the context and project, but there are some easy steps that can help make the most of working with enumerators.

\begin{tabular}{|c|c|}
\hline Do & Don't \\
\hline $\begin{array}{l}\text { Plan sufficient time for getting to know and } \\
\text { training enumerators. }\end{array}$ & $\begin{array}{l}\text { Don't cut corners by reducing the time allocated } \\
\text { to training. }\end{array}$ \\
\hline $\begin{array}{l}\text { Make sure that when starting to train enumerators } \\
\text { for a project, you have considered the necessary } \\
\text { buy-in from relevant people. }\end{array}$ & $\begin{array}{l}\text { Don't assume the benefits of the research you } \\
\text { are conducting will be known and/or obvious to } \\
\text { everyone. }\end{array}$ \\
\hline $\begin{array}{l}\text { Ensure you allow for sufficient flexibility in the time } \\
\text { allocated to training - including time for piloting } \\
\text { the research tools and any adjustments that need } \\
\text { to be made as a result. }\end{array}$ & $\begin{array}{l}\text { Don't expect that just telling enumerators what } \\
\text { to do is enough to ensure they collect data at } \\
\text { the expected level of quality. }\end{array}$ \\
\hline $\begin{array}{l}\text { Include role-plays in your training, in which two or } \\
\text { more enumerators demonstrate best and worst } \\
\text { case interview scenarios - repeating this will help } \\
\text { turn theory into practice, and reveal the extent to } \\
\text { which enumerators understand what is expected } \\
\text { of them. }\end{array}$ & $\begin{array}{l}\text { Don't rely exclusively on questions raised by } \\
\text { enumerators to understand whether something } \\
\text { they are expected to do is clear - there are } \\
\text { many reasons why people hold back, } \\
\text { particularly in front of a group. }\end{array}$ \\
\hline $\begin{array}{l}\text { Give constructive and individualized feedback } \\
\text { throughout, including during the piloting stage. }\end{array}$ & $\begin{array}{l}\text { Don't expect that giving feedback once will be } \\
\text { enough to change a habit or approach that an } \\
\text { enumerator may have. }\end{array}$ \\
\hline $\begin{array}{l}\text { Allocate enough time to understand and practice } \\
\text { obtaining truly informed consent from } \\
\text { respondents as a crucial part of high quality data } \\
\text { collection. }\end{array}$ & $\begin{array}{l}\text { Don't assume enumerators are aware why } \\
\text { informed consent is important or what } \\
\text { constitutes a sufficient level of 'informed'. As } \\
\text { safeguarding protocols are being tightened } \\
\text { throughout the development sector (see the } \\
\text { section on research ethics below), obtaining } \\
\text { informed consent is critical. }\end{array}$ \\
\hline $\begin{array}{l}\text { Clarify benchmarks for what is considered high } \\
\text { quality data, and how you will implement a data } \\
\text { quality control protocol. Explain what the } \\
\text { feedback process on enumerator performance will } \\
\text { look like once data collection starts, and what } \\
\text { consequences for poor data collection will ensue. }\end{array}$ & $\begin{array}{l}\text { Don't expect that everyone in your enumerator } \\
\text { group will deliver similar data quality (even if } \\
\text { their skills appear similar during training), or that } \\
\text { quality will remain constant throughout data } \\
\text { collection, especially when it lasts for a longer } \\
\text { time period. }\end{array}$ \\
\hline
\end{tabular}




\section{DATA COLLECTION}

\section{Enumerators and research ethics}

Enumerators need to be aware of and comply with ethical standards of research, which have to be communicated from the start of the research. For example, enumerators working for Oxfam must sign Oxfam's Code of Conduct and Data Handling agreement, which must be talked through during their training so they genuinely understand the implications and their responsibilities and rights.

This includes but is not limited to the following areas:

- Treat respondents with respect and dignity, and act on the principle of doing no harm. This means the benefits of the research must outweigh the risks, implying enumerators need to be able to manage respondents' emotions and deal with unexpected challenges during the fieldwork.

- Understand informed consent: what it is, how to ask for and obtain it from participants, and how to record it alongside the information being collected for data protection purposes. Obtaining consent is more than following a standard template, it involves an open conversation, the ability to ask questions, and is very contextual. Basic templates to guide obtaining informed consent are readily available from sources like the UK Data Service and Oxfam's Mobile Survey Toolkit.

- Even if collected elsewhere, if it is being stored inside the EU, personal data is closely bounded by EU General Data Protection Regulation (GDPR) guidelines. The GDPR limits what kind of personal data organizations and companies can hold, for how long and under what security and privacy conditions. Enumerators and any potential supervisors need to understand the importance of respondents' right to privacy and knowledge of what happens to their personal data. This includes knowing what data should and should not kept together or separately on what servers, devices or physical places.

- Be aware of the types of cases of abuse, events that may be illegal or warrant a safeguarding investigation which have been uncovered through data collection that need to be reported to the appropriate superiors or services such as EthicsPoint. Responsibilities and referrals for this process should be clarified at the outset of each project.

At the same time, whoever contracts enumerators has a duty of care towards them. This means being aware that they may be exposed to information that is particularly difficult to digest or that may result in trauma, and having a protocol that enumerators can follow if they have difficulty coping with such information. Ensuring enumerators are supported at the end of each day of data collection can help them process potentially difficult conversations or encounters.

\section{Survey error and data quality}

There are many ways in which quantitative and qualitative data can be biased by enumerators and respondents. For an overview, see Oxfam's research guidelines on Planning Survey Research and Understanding Survey Sampling. To improve the collection of digital household survey data in particular, see Oxfam's Going Digital series. The following list is not exhaustive, but covers some of the most common survey error and data quality problems involvling enumerators.

\section{Obtaining informed consent}

Enumerators often cut or skip conversations that they should have to obtain informed consent, leaving the forms blank or forging information. For smaller scale data collection, especially on paper, this can be uncovered by accompanying and monitoring enumerators, or - participant 
consent permitting - asking intermediaries to do so and to request audio or video footage of how interviews were conducted. In digital data collection, consent forms can be pre-recorded as audio files on the data collection device, and playing this file can be marked as mandatory.

\section{Changing the meaning of questions}

Eumerators may ask questions differently than intended in the research. This can be for a range of reasons, for example, misunderstanding the intended meaning due to insufficient training or oversight. Questions may also be poorly worded or translated, or may not make much sense in context. Enumerators may also shorten them to save time, especially when working towards a quota of interviews that have to be completed in a certain amount of time, thereby losing some of the original meaning. In short, this problem can come in many forms, and as above, the best course of action is to implement a context-specific data quality control protocol.

\section{Saving time by cutting corners}

Sometimes enumerators 'learn' that certain responses allow them to save substantial amounts of time, so they tend to choose those answers -- either in place of the respondent, through rewording the question, or both. Audio or video recordings that show how enumerators collect data at regular or random moments can be requested, or enumerators accompanied. During digital data collection, consistency checks can be programmed to automatically flag problematic interviewer patterns and behaviours - for example, where sections are skipped, questions always answered the same way, where they take too little or too long to be answered, or where answers from one section do not logically translate into other information.

\section{Spotting social desirability and other biases}

Social desirability bias leads respondents to disclose the kind of information they think is expected of them and/or socially appropriate. Enumerators can play their part in minimizing the risk that this bias will systematically distort the data. To ensure respondents feel free and empowered to speak to their own experience, the risk of this bias should be discussed during training and appropriate responses practised. Piloting is also crucial to think about possible solutions, as is controlling enumerator performance.

Through the information they share, repondents might also try to get access to resources - again, enumerators can play their part to minimize this risk. For example, when aid agencies collect data from vulnerable people on how well an assistance programme has performed, (potential) beneficiaries are more likely to speak favourably in order to increase their chances of getting help. This type of bias may be addressed by stakeholder discussions, for example by involving community leaders who can explain the importance of unbiased data collection to respondents. 


\section{WORKING WITH ENUMERATORS: A CHECKLIST}

The following list summarizes some of the key decisions that need to be considered when doing research with enumerators, in order to collect meaningful data of the highest possible quality.

- What is the profile of the enumerators I should be looking for? What, if any, identity and socioeconomic factors do I need to be mindful of?

- Does my project rely on recruiting and managing enumerators directly, or through an intermediary? If so, are the incentives for each person or team and their responsibilities clear?

- Are enumerators aware from the start of the project what their value-added is, what their exact responsibilities are and how they will be remunerated and managed?

- How many days should I plan for training enumerators, piloting the research tool(s) and pretesting interviews, and what does this mean for my budget?

- How many interviews per day or week can I expect from each enumerator, and is the expectation clear for an achieved quota and high quality data?

- Do enumerators understand the ethical implications of the research they are involved in, as well as the risk of bias and the potential for low quality data that comes with poor administration of whatever tool is being used?

- Do I have a protocol in place to monitor quality and to deal with data quality red flags?

\section{ADDITIONAL RESOURCES}

You might also want to check out other Oxfam research quidelines and Research in Practice case studies before you start planning research.

\section{Links}

All links last accessed October 2018.

Oxfam's Effectiveness Reviews: https://policy-practice.oxfam.org.uk/our-approach/monitoringevaluation/effectiveness-reviews

How Decent is Decent Work? Using SenseMaker to understand workers' experiences https://policy-practice.oxfam.org.uk/publications/how-decent-is-decent-work-using-sensemaker-tounderstand-workers-experiences-620476

Going Digital: Improving data quality with digital data collection https://policy-practice.oxfam.org.uk/publications/going-digital-improving-data-quality-with-digitaldata-collection-620522

Agile research in the risky realities of the Central African Republic https://views-voices.oxfam.org.uk/methodology/real-geek/2017/06/agile-research-car

UK Data Service Consent for data sharing: https://www.ukdataservice.ac.uk/manage-data/legalethical/consent-data-sharing/consent-forms 
Mobile Survey Toolkit

https://policy-practice.oxfam.org.uk/publications/mobile-survey-toolkit-617456

EU General Data Protection Regulation (GDPR): https://eugdpr.org/

EthicsPoint: https://secure.ethicspoint.eu/domain/media/en/gui/104806/

Planning Survey Research

https://policy-practice.oxfam.org.uk/publications/planning-survey-research-578973

Understanding Survey Sampling

https://policy-practice.oxfam.org.uk/publications/understanding-survey-sampling-615904

Going Digital series: https://policy-practice.oxfam.org.uk/publications/search?q=going digital

Improving data quality with digital data collection

https://views-voices.oxfam.org.uk/methodology/2018/07/improving-data-quality-with-digital-data-

collection

Oxfam research guidelines: https://policy-practice.oxfam.org.uk/our-approach/research/researchguidelines\#d45f0f19-056e-4b9c-9a00-bbad4d81be78

Oxfam Research in Practice case studies: https://policy-practice.oxfam.org.uk/ourapproach/research/research-guidelines\#a9913f92-0991-4139-836d-871190df9d1a

(C) Oxfam International November 2018

This guideline has been prepared by Oxfam for use by development practitioners and researchers, including Oxfam staff and partners. It was written by Franziska Mager and Amy Croome and edited by Martin Walsh from Oxfam GB's Research Team, with thanks to Andrea Azevedo, Irene Guijt, Simone Lombardini, Emily Tomkys Valteri and Jaynie Whinnery for their inputs.

The text may be used free of charge for the purposes of education and research, provided that the source is acknowledged in full. The copyright holder requests that all such use be registered with them for impact assessment purposes. For copying in any other circumstances, or for re-use in other publications, or for translation or adaptation, permission must be secured and a fee may be charged. Email publish@oxfam.org.uk

Oxfam welcomes comments and feedback on its Research Guidelines. If you would would like to discuss any aspect of this document, please contact research@oxfam.org.uk. For further information on Oxfam's research and publications, please visit www.oxfam.org.uk/policyandpractice

The information in this publication is correct at the time of going to press.

Published by Oxfam GB for Oxfam International under ISBN 978-1-78748-357-6 in November 2018. DOI: $10.21201 / 2018.3576$

Oxfam GB, Oxfam House, John Smith Drive, Cowley, Oxford, OX4 2JY, UK. Oxfam is a registered charity in England and Wales (no 202918) and Scotland (SC039042).

Oxfam is an international confederation of 19 affiliates networked together in more than 90 countries, as part of a global movement for change, to build a future free from the injustice of poverty.

www.oxfam.org 